\title{
Retrieval Of GammaCell 220 Irradiator Isodose Curves With MCNP Simulations And Experimental Measurements
}

\author{
R.R. Rodrigues, ${ }^{*}$ S.E. Grynberg, A.V. Ferreira ${ }^{\dagger}$ L.C.M. Belo, ${ }^{\ddagger}$ and P.L. Squair ${ }^{\S}$ \\ Centro de Desenvolvimento da Tecnologia Nuclear (CDTN), \\ Av. Presidente Antônio Carlos, 6627 - Caixa Postal 941, \\ Pampulha, Belo Horizonte, MG, CEP 31270-901, Brasil \\ R.V. Sousa ${ }^{\mathbb{T l}}$ and R.C.O. Sebastião** \\ Universidade Federal de Minas Gerais (UFMG), Instituto de Ciências Exatas - Departamento de Química \\ M.A. Ribeiro ${ }^{\dagger \dagger}$ \\ Centro de Desenvolvimento da Tecnologia Nuclear (CDTN), \\ Av. Presidente Antônio Carlos, 6627 - Caixa Postal 941, \\ Pampulha, Belo Horizonte, MG, CEP 31270-901, Brasil and \\ Universidade Federal de Minas Gerais (UFMG), Instituto de Ciências Exatas - Escola de Engenharia Química, \\ Av. Presidente Antônio Carlos, 6627, Pampulha, Belo Horizonte, MG, CEP 31270-901, Brasil \\ (Received on 29 January, 2010)

\begin{abstract}
Gamma irradiator facilities can be used in a wide range of applications such as biological and chemical researches, sterilization of medical devices and products. Dose mapping must be performed in these equipments in order to establish plant operational parameters, as dose uniformity, source utilization efficiency and maximum and minimum dose positions. The isodoses curves are measured using dosimeters or computer simulations. This work evaluates the absorbed dose in the CDTN/CNEN GammaCell Irradiation Facility, using the Monte Carlo N-Particles (MCNP) code.

Keywords: Monte Carlo; GammaCell 220; Isodose; Dosimetry; MCNP
\end{abstract}

\section{INTRODUCTION}

The industrial irradiator GammaCell 220 was originally manufactured by Atomic Energy of Canada Limited (AECL) which is now part of MDS Nordion International. It was developed for high dose rate gamma radiation researches and studies. It can be used in a wide variety of research areas, like food irradiation, geology, medical area, material research, radiation effects on biological systems, sterilization of organisms and soils, changing and improving properties of polymers, and others. This equipment uses ${ }^{60} \mathrm{Co}$ doubly encapsulated in aluminum and stainless steel tubes, fixed within a lead shield, and the operator is not exposed to the emitted radiation. The GammaCell exposure chamber has a cylindrical geometry with $20.3 \mathrm{~cm}$ height and $7.5 \mathrm{~cm}$ radius. The manufacturer's calibration certificate states a dose rate of $(4.43 \pm$ $0.16) \times 10^{3} \mathrm{~Gy} / \mathrm{h}$ on $08 / 28 / 1962$ in the center of the exposure chamber. Figure 1 shows the isodoses provided by the manufacturer in 1962 [1].

Isodose curves are generally measured using dosimeters distributed throughout the device. This procedure consumes a large amount of dosimeters, irradiation time and manpower [2]. The use of mathematical methods and radiation transport codes can reduce significantly this problem, although complex and time-consuming codes might be neces-

\footnotetext{
*Electronic address: rrrecdtn.br

${ }^{\dagger}$ Electronic address: avfecdtn.br

¥Electronic address: 1 cmb@cdtn.br

§lectronic address: pls@cdtn.br

TElectronic address: romuloverdolin@yahoo.com.br

** Electronic address: ritacoseufmg.br

${ }^{\dagger}$ Electronic address: marianaalmeida@ufmg.br
}

sary $[3,4]$. Monte Carlo methods make possible to calculate various parameters like flux, fluence, energy spectra and absorbed dose [1,5-7].

The Monte Carlo code requires an input file with information such as the geometry specification, description of materials, selection of cross-section reactions, location and characteristics of the radiation source, type of answers desired and variance reduction techniques $[2,5,8]$. The aim of this work is to perform a dose mapping simulation for the CDTN/CNEN GammaCell 220 using this code. The results of the simulation will be validated with experimental results obtained by FRICKE and TLD dosimetry.

\section{METHODOLOGY}

\section{The MCNP Code}

The MCNP is a general-purpose Monte Carlo N-Particle code that can be used for various types of ionizing radiations like neutron, photon and electron, among others. An input file created by the user is read by MCNP. It contains informations about the problem in areas such as the geometry specification; the description of materials and selection of cross-section evaluations; the location and characteristics of the neutron, photon, or electron source; the type of answers or tallies desired; and any variance reduction techniques used to improve efficiency [5].

MCNP is useful for complex problems that cannot be modeled by other computer codes using deterministic methods. This code simulates sequentially the individual probabilistic events that comprise a process. These probability distributions are statistically sampled to describe the total phenomenon. The number of trials necessary to describe a phe- 


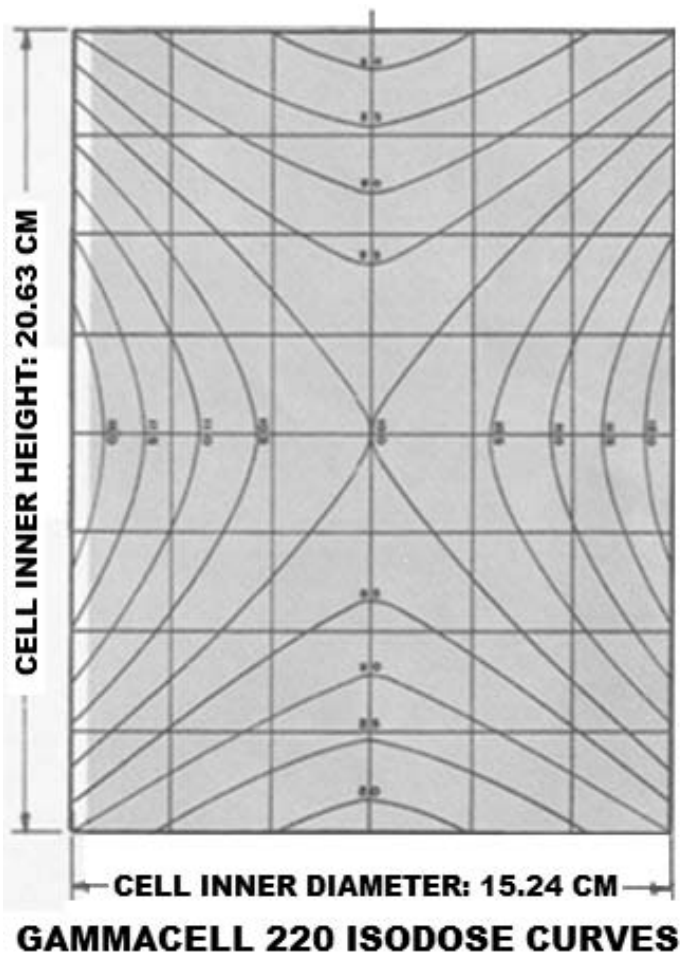

FIG. 1: GammaCell 220 certified isodoses.

nomenon is quite large and a powerful computer has to be used for the simulations. The statistical sampling process is based on the selection of random numbers. The code follows each of many particles from a source throughout its life to its death, considering all the probable events like absorption and escape.

It is necessary to associate nuclear data tables with this code. There are nine classes of data tables for MCNP:

- continuous-energy neutron interaction data;

- discrete reaction neutron interaction data;

- continuous-energy photoatomic interaction data;

- continuous-energy photonuclear interaction data;

- neutron dosimetry cross sections;

- neutron $S(\alpha, \beta)$ thermal data;

- multigroup neutron, coupled neutron/photon, and charged particles masquerading as neutrons;

- multigroup photon;

- electron interaction data [5].

The available tally cards provide these informations: surface current; surface flux; track length estimate of cell flux; flux at a point (point detector); track length estimate of energy deposition; track length estimate of fission energy deposition; and energy distribution of pulses created in a detector.

\section{The MCNP Simulations}

The simulations of the industrial irradiator GammaCell 220 were carried out by the MCNP-4B radiation transport code. The irradiator geometry was simulated as closely as possible to reproduce the experimental setup. Figure 2 shows the simulated geometry.
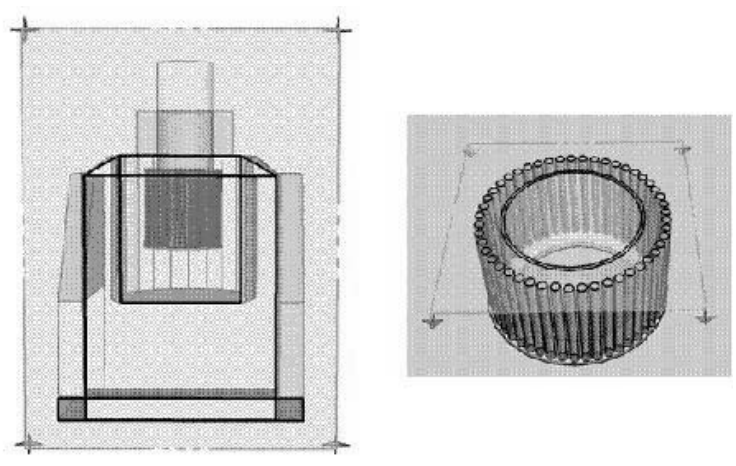

FIG. 2: Frontal cut-away view of GammaCell 220 and top view of sources.

The input file contains 240 surfaces and 90 cells. There are $54{ }^{60} \mathrm{Co}$ cylindrical sources of $0.3594 \mathrm{~cm}$ radius and 24.6276 $\mathrm{cm}$ length doubly encapsulated in aluminum and stainless steel tubes. Radioactive decay was taken into account in the simulation, and nickel concentration was introduced in the material definition of the sources. The cylindrical sources are separated from the exposure chamber by an aluminum and stainless steel shell of $0.2 \mathrm{~cm}$ thick with 7.5 and $7.7 \mathrm{~cm}$ inner radius, respectively. Outside the sources, a structure constituted by a lead shield was designed.

The sources were simulated using the sdef card, where the photon emission starting points are centered in the 54 cylinders. The extension and emission spectrum was defined as a Maxwellian distribution, appropriated for this problem, together with the efficiency specification. The two energy peaks of ${ }^{60} \mathrm{Co}$ : 1.17321 and $1.332491 \mathrm{MeV}$ with 0.9986 and 0.9998 probabilities were also considered in the simulation.

The tally cards used were F4 (flux in a cell, measured in photons $/ \mathrm{cm}^{2}$ ), being modified by a dose function using the $d e$ and $d f$ cards. These tallied quantities, $\mathrm{C}$, can be represented as

$$
C=\int \varphi(E) f(E) d E
$$

with $\varphi(E)$ the energy-dependent fluency and $f(E)$ the product quantities in the cross-section libraries. The ring detectors are positioned at half height and a bigger importance in the central detectors was also adopted.

\section{Experimental Dosimetry}

\section{a) Thermoluminescent Dosimetry}

In this work, a disc shape acrylic sample-holder was constructed to accommodate 49 TLD-800, as shown in Figure 3. 
Each TLD crystal has approximately $3 \times 3 \times 1 \mathrm{~mm}^{3}$. The sample-holder was loaded with the TLD's and accommodated in the GammaCell exposure chamber at half height. The set was irradiated with a nominal dose of $4.0 \mathrm{~Gy}$. The nominal dose value in the experiments corresponds to the expected dose calculated with the certificated dose rate and the radioactive decay law. The TLD's analyses were done in a Harshaw TLD reader model 4500 . This entire procedure was repeated four times.
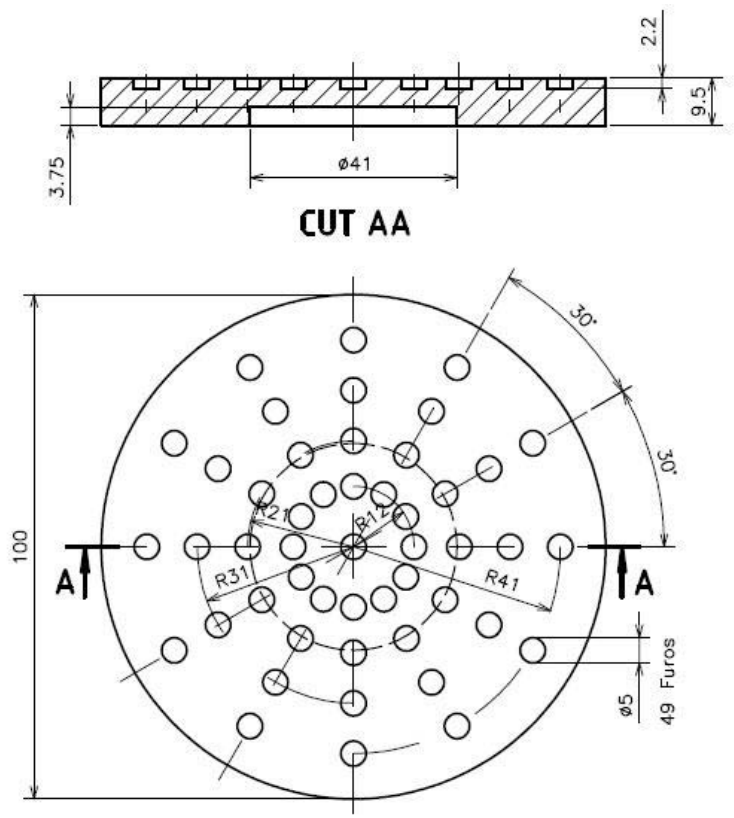

FIG. 3: TLD's sample-holder diagram.

\section{b) Fricke Dosimeter}

The Fricke dosimeter is a chemical dosimeter consisting of an aqueous solution of sulfuric acid and ferrous sulfate in the following composition: $1 \mathrm{mM} \mathrm{FeSO}_{4}+0.8 \mathrm{~N} \mathrm{H}_{2} \mathrm{SO}_{4}+$ $1 \mathrm{mM} \mathrm{NaCl}$. The irradiation of the Fricke solution causes radiolysis of water and as a secondary reaction, oxidize the ferrous ions to ferric ions, which in turn have a strong optical absorption with peaks at wavelengths $224 \mathrm{~nm}$ and $304 \mathrm{~nm}$ [9]. The Fricke dosimeter is an absolute dosimeter (requires no calibration) and the absorbed dose is determined from the yield of ferrous ions, determined by spectrophotometers. This study used a Shimadzu UV1240 spectrophotometer. The Fricke dosimeters were placed in cylindrical glass containers with internal dimensions of $40 \mathrm{~mm}$ in height and $10 \mathrm{~mm}$ in diameter. Five measurements were performed with the dosimeters placed in the central portion of the chamber, with nominal doses of 50, 90, 150, 200 and 250 Gy. Subsequently seventeen samples were irradiated in a circular arrangement at the half height: eight samples were placed at maximum radius of the exposure chamber, other eight in half of the radius, and one in the center. This sample set was irradiated with nominal dose of $160 \mathrm{~Gy}$.

\section{RESULTS AND DISCUSSIONS}

\section{Thermoluminescent Dosimetry}

The dose results measured using TLD's are shown in Figure 4. In this representation the doses were normalized in relation to the dose measured at the central position. The TLD's results indicate that along the $\mathrm{B}$ direction there is a greater symmetry in the doses distribution than along the A direction. The observed asymmetry was not expected considering the isodose curves supplied by the equipment manufacturer (see Fig. 1).

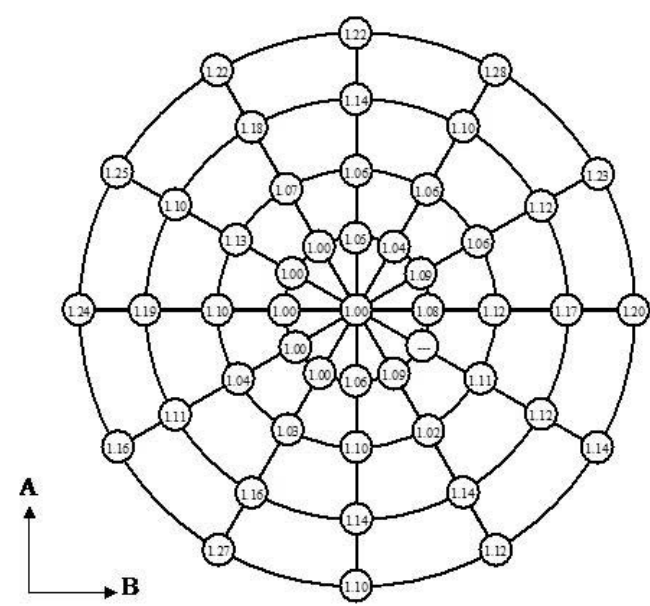

FIG. 4: TLD experimental doses

\section{FRICKE Dosimetry}

The results obtained with the Fricke dosimeters, at the central volume of the irradiation chamber for different doses are shown in Figure 5. The dependence observed between the evaluated doses and the nominal doses is linear, with evaluated values $7 \%$ higher than nominal values in average.

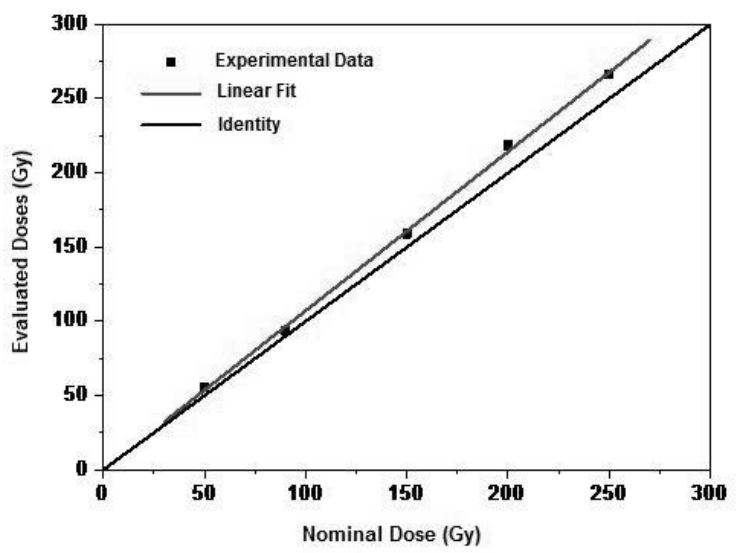

FIG. 5: Measured doses for GammaCell 220 with Fricke dosimeters positioned at the center of the exposure chamber

The results obtained for the circular arrangement with a 
nominal dose of 160 Gy are shown in Figure 6.

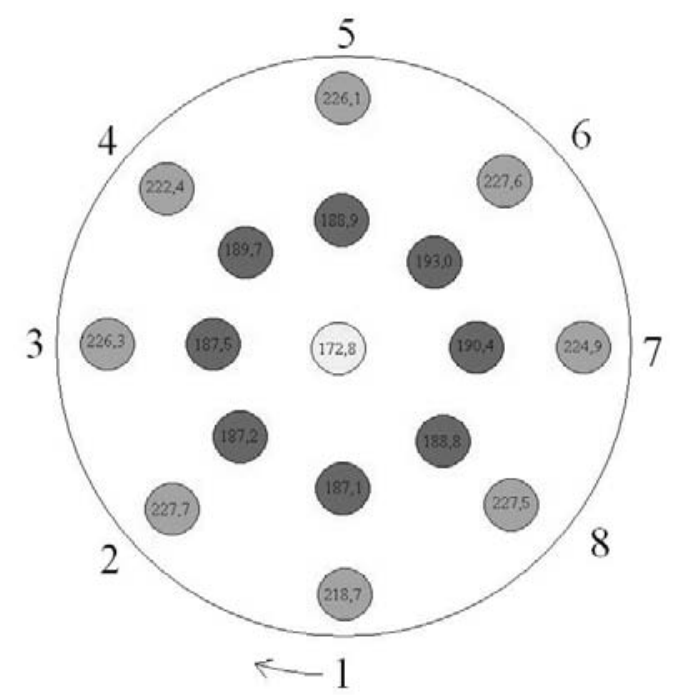

FIG. 6: Experimental doses at the plan in the half height of GammaCell 220 exposure chamber for a 160 Gy nominal dose

From Figure 6, it is observed that at the center, the measured dose 172.8 Gy is $8 \%$ higher than the nominal dose 160 Gy, which agrees with the results showed in Figure 5. The mean measured values for gamma doses at half radius and radius are $(189 \pm 2)$ Gy and (225 \pm 3$)$ Gy respectively. These values are $9 \%$ and $17 \%$ higher than the expected ones (173 and 192 Gy) according to the Certificate and the decay law. These results are resumed at Table 1 .

The difference between the expected and the measured doses increases with the radius. This behavior is not well understood yet. It indicates the need of performing further experiments to verify if there is a systematic measurement error or if there is a real difference between the delivered doses and the certified ones.

In the representation of Figure 6 the doses were not normalized in relation to the dose measured at the central position. When the normalization is done, the dose delivered at half radius is $109 \%$ while the dose at radius is $130 \%$.

\section{Monte Carlo Simulations}

Isodose curves simulation of the GammaCell 220 irradiator was performed using ring detectors with $1 \mathrm{~cm}$ height, $0.5 \mathrm{~cm}$ thickness and variable radius, as presented in Table 2. They were positioned at half height in the exposure chamber and $1 \times 10^{6}$ particle histories were considered in the simulation. The relative doses with respect to the central point are also presented in Table 2.

The accuracy of the MCNP calculations is mainly related to the input and can be verified by the experimental relative errors. For these ring detector positions, the evaluated relative errors in comparison with the GammaCell 220 certified isodoses curves (1962) are presented in Table 3.

A valid confidence interval for dose calculation can be represented by a smaller value of standard deviation, $\mathrm{S}$, and variance $S x$, which is evaluated in its usual meaning. The variance
TABLE 1: Comparison of nominal doses and measured doses.

\begin{tabular}{cllll}
$\begin{array}{c}\text { Delivered } \\
\text { Dose } \\
\text { (cGy) }\end{array}$ & $\begin{array}{c}\text { Posi- } \\
\text { tion }\end{array}$ & $\begin{array}{c}\text { Nominal } \\
\text { Dose (cGy) }\end{array}$ & $\begin{array}{c}\text { Measured } \\
\text { Dose (cGy) }\end{array}$ & $\begin{array}{c}\text { Diffe } \\
\text { rence } \\
\text { (\%) }\end{array}$ \\
\hline \multirow{3}{*}{160} & Center & 160 & 172.8 & 8 \\
& $\mathrm{R} / 2$ & 173 & $189 \pm 2$ & 9 \\
& $\mathrm{R}$ & 192 & $225 \pm 3$ & 17
\end{tabular}

TABLE 2: Ring detector radius and simulated relative doses.

\begin{tabular}{cc} 
Ring detector radius $(\mathrm{cm})$ & Dose $(\%)$ \\
\hline 0.25 & 100 \\
0.75 & 102 \\
1.25 & 103 \\
2.75 & 104 \\
3.25 & 106 \\
3.75 & 108 \\
4.25 & 109 \\
4.75 & 113 \\
5.75 & 117 \\
6.25 & 119 \\
6.75 & 123
\end{tabular}

TABLE 3: Certified and Simulated GammaCell 220 isodoses comparison.

\begin{tabular}{cccc} 
Radius (cm) & $\begin{array}{c}\text { Certified } \\
\text { isodoses - } \\
1962(\%)\end{array}$ & $\begin{array}{c}\text { Simulated } \\
\text { dose (\%) }\end{array}$ & $\begin{array}{c}\text { Relative } \\
\text { error } \\
(\%)\end{array}$ \\
\hline 3.00 & 105 & 104 & 0.95 \\
4.55 & 110 & 109 & 0.90 \\
5.81 & 115 & 117 & 1.74 \\
6.90 & 120 & 123 & 2.50
\end{tabular}

is a convenient parameter to represent statistical accuracy. It was empirically verified in MCNP calculations that it should be less than 0.1 for reliable detectors. This value takes into account the contributions near to the detectors that are usually extremely important and difficult to sample. The variance evaluated for dose curves in each position is acceptable for this problem, being less than 0.05 for all detectors.

The MCNP simulated results were also compared with the Fricke and TLD results. Figure 7 plots values for the GammaCell 220 certified isodoses from 1962 and the MCNP, TLD's and Fricke normalized doses in function of the radius.

The TLD's and Fricke dosimetry were also verified in the present work. Both were tested and compared to the GammaCell 220 certified isodoses (1962). The TLD's and FRICKE dosimeters indicated incoherent relative doses in comparison with simulated results, with relative errors varying from 1 to 9\%. This result is neither in agreement with the GammaCell certificate and further studies have to be performed to understand this experimental behavior. Nevertheless, the good agreement in the simulated and certified results indicates the potentiality of the simulation method. 


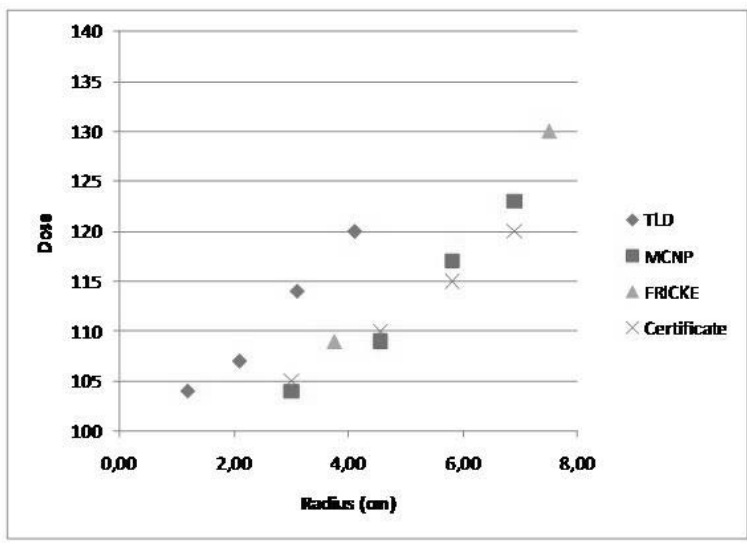

FIG. 7: GammaCell 220 certified isodoses and the MCNP, TLDs and FRICKE normalized doses in function of the radius

\section{CONCLUSIONS}

In this work experimental and simulated isodoses of GammaCell 220 was obtained. The simulation was performed using the Monte Carlo methodology and the experimental curves were obtained by GammaCell 220 Certificate, Thermoluminescent and FRICKE dosimeters. The simulation results showed a good agreement with the GammaCell 220 certificates, and except in one position (with relative error of $9 \%$ ), the relative error with the experimental data is less than $3 \%$, indicating that the simulation methodology can be used as a routine predictive tool of irradiation planning.

Dose evaluation is generally referred to a mass of air that occupies the entire chamber of gamma irradiator facilities. This routine does not take into account the peculiarities of each sample, especially with respect to volume, composition and density, which are essential parameters to determine the actual accumulated energy dose. The MCNP code developed here can also contribute in this sense, bringing a significant improvement in the irradiation planning.

\section{Acknowledgments}

Work supported by the Minas Gerais State FAPEMIG (Fundação de Amparo a Pesquisa do Estado de Minas Gerais).
[1] M. Sohrabpour, M. Hassanzadeh, M. Shahriari, M. Sharifzadeh, Gamma irradiator dose mapping simulation using the MCNP code and benchmarking with dosimetry. Applied Radiation and Isotopes 57, pp. 537-542, 2002.

[2] C. Oliveira, J. Salgado, M. Luísa Botelho, L.M.Ferreira. Dose determination by Monte Carlo - a useful tool in gamma radiation process. Radiation Physics and Chemistry 57, pp. 667-670, 2000.

[3] G. Piña-Villalpando and D.P. Sloan, Dose distribution studies of a gamma industrial irradiator using a PC code. Radiation Physics and Chemistry 52, Nos. 1-6, pp. 563-567, Great Britain, 1998.

[4] C. Oliveira, J. Salgado, M. Luísa Botelho, L.M. Ferreira. Monte Carlo studies for irradiation process planning at the Portuguese gamma irradiation facility. Applied Radiation and Isotopes 53, pp. 867-875, USA, 2000.

[5] X-5 Monte Carlo Team. MCNP - A General Monte Carlo NParticle Transport Code, Version 5. Volume I - Overview and
Theory. Los Angeles National Laboratory, University of California, USA, 2003.

[6] C. Oliveira, J. Salgado. Isodose distributions and dose uniformity in the Portuguese gamma irradiation facility calculated using the MCNP code. Radiation Physics and Chemistry 61, pp. 791-793, 2001.

[7] Weiss, Douglas E.; Johnson, William C.; Kensek, Ronald P. Dose distributions in tubing irradiated by electron beam: Monte Carlo simulation and measurement. Radiation Physics and Chemistry 50, No. 5, pp. 475-485, Great Britain, 1997.

[8] C. Oliveira, J. Salgado, A. Ferro de Carvalho. Dose rate determinations in the Portuguese Gamma Irradiation Facility: Monte Carlo simulations and measurements. Radiation Physics and Chemistry 58, pp. 279-285, 2000.

[9] M.A. Ribeiro, A.V. Ferreira, L.C. Meira-Belo, S.E. Grynberg. Use of FRICKE dosimeters for the evaluation of gamma fields. INAC 2009. 